\title{
Column extraction of heavy metals from soils using the biodegradable chelating agent EDDS
}

\author{
Lukas Hauser, Susan Tandy, Rainer Schulin, and Bernd Nowack*
}

\author{
Institute of Terrestrial Ecology (ITÖ) \\ Swiss Federal Institute of Technology Zürich (ETH) \\ Grabenstrasse 3 \\ CH-8952 Schlieren, Switzerland \\ Phone: +41 (0)44 6336160 \\ Fax: +41 (0)446331123 \\ e-mail: nowack@env.ethz.ch
}

\section{Supporting Information}

Table 1S: Comparison of the batch and column extraction efficiency for $\mathrm{Fe}, \mathrm{Ca}$, and $\mathrm{Mg}$ 
Table 1S: Comparison of the batch and column extraction efficiency for $\mathrm{Fe}, \mathrm{Ca}$, and $\mathrm{Mg}$ (average \pm standard deviation). Ratio chelant:metals $=1$. Batch data are for a soil:solution ratio of 1:2, pH 7.5 and tap water except where noted.

\begin{tabular}{|c|c|c|c|c|c|}
\hline \multirow[t]{2}{*}{ Metal } & \multirow[t]{2}{*}{ soil } & \multirow[t]{2}{*}{ metal:chelant } & batch & $\begin{array}{l}\text { column } \\
\text { low } \\
\text { infiltration }\end{array}$ & \multirow[t]{2}{*}{$\begin{array}{l}\text { column } \\
\text { fast } \\
\text { infiltration }\end{array}$} \\
\hline & & & & $\mathrm{mg} \mathrm{kg}^{-1}$ & \\
\hline \multirow[t]{4}{*}{$\mathrm{Fe}$} & Dornach 1 & 1 & $115 \pm 5$ & $480 \pm 96$ & \multirow{4}{*}{$78 \pm 19$} \\
\hline & Dornach 2 & 1 & $194 \pm 13$ & $408 \pm 35$ & \\
\hline & Rafz & 1 & $147 \pm 2$ & $180 \pm 6$ & \\
\hline & Rafz & 10 & $370 \pm^{1)}$ & $711 \pm 21$ & \\
\hline \multirow[t]{4}{*}{$\mathrm{Ca}$} & Dornach 1 & 1 & $517 \pm 51$ & $450 \pm 62$ & \\
\hline & Dornach 2 & 1 & $90 \pm 23$ & $497 \pm 90$ & \\
\hline & Rafz & 1 & $-2 \pm 18$ & $232 \pm 13$ & \\
\hline & Rafz & 10 & na & $1932 \pm 713$ & \\
\hline \multirow[t]{4}{*}{$\mathrm{Mg}$} & Dornach 1 & 1 & $-6 \pm 0.4$ & $18 \pm 2$ & \\
\hline & Dornach 2 & 1 & $-18 \pm 1$ & $28 \pm 2$ & \\
\hline & Rafz & 1 & $17 \pm 15$ & $29 \pm 2$ & \\
\hline & Rafz & 10 & na & $142 \pm 12$ & \\
\hline
\end{tabular}

na: not available

1) Solid: solution ratio 1:50, ratio chelant: metal: $10, \mathrm{pH} 7.5$ - 8, tap water 